\title{
La metodología de clase inversa en la UPV: Una experiencia en el laboratorio de matemáticas
}

\author{
Carmen Coll, Damián Ginestar, Esther Sanabria y Elena Sánchez \\ Departamento de Matemática Aplicada, Universitat Politècnica de València \\ (ㅆcoll@mat.upv.es,dginesta@mat.upv.es, esanabri@mat.upv.es y esanchezj@mat.upv.es)
}

\begin{abstract}
In this paper, we analyze the experience of introducing the flip teaching methodology in the laboratory practices of Mathematics II. In this subject our students learn differential equations, Laplace transform and their applications, concepts that a priori are not too attractive to students of engineering degrees, but are very useful in the development of different subjects that are part of their training. In order to make the students more motivated, we have redesigned the activities carried out in Mathematics II practices, preparing several materials and strategies that activate the interest of the students. Some of these materials are available before practice sessions and others, such as problem-solving, discussion or debates, are used in the classroom itself at the time of practice. The main novelty is the screencast videos we have recorded, where we explain how to approach the different types of exercises that we solve using the program Mathematica. This increases student opportunities for active learning in class, where the teacher can offer a more personalized guidance and interact better with the students. The interaction with the teacher and the other classmates generates an environment that facilitates learning, as we have seen through specific evaluation tests.
\end{abstract}

Keywords: Flip teaching (or flipped classroom), methodology, evaluation, active participation.

\footnotetext{
Resumen

En este trabajo analizamos la experiencia de introducir la metodología denominada clase inversa (o 'flip education') en las prácticas de laboratorio de Matemáticas II. En esta asignatura trabajamos ecuaciones diferenciales, transformada de Laplace y sus aplicaciones, conceptos que a priori no resultan demasiado atractivos para los alumnos de los grados de ingeniería, pero resultan de gran utilidad en el desarrollo de las distintas materias que forman parte de su titulación. Con el fin de conseguir una mayor motivación entre nuestro alumnado, hemos utilizando la metodología de clase inversa en las prácticas de Matemáticas II, rediseñado las actividades realizadas en dicha asignatura y preparado materiales que activen el interés de los alumnos.
} 
La principal novedad son los vídeos 'screencast' que hemos grabado, donde explicamos cómo abordar los distintos tipos de ejercicios que trabajamos utilizando el programa Mathematica, de manera que el tiempo que los alumnos pasen en el laboratorio con el profesor lo aprovechen para resolver las dudas que les hayan surgido y aclarar los conceptos principales de cada sesión. Esta interacción con el profesor y el resto de compañeros genera en el aula un ambiente que facilita el aprendizaje, como hemos comprobado mediante pruebas de evaluación especificas.

Palabras clave: clase inversa, metodología, evaluación, participación activa.

\section{Introducción}

Las ecuaciones diferenciales siempre han formado parte de los contenidos de las asignaturas de matemáticas de todas las ingenierías, en particular, de las impartidas en las titulaciones de la Escuela Técnica Superior de Ingeniería del Diseño (ETSID) de la Universitat Politècnica de València (UPV). Actualmente, estos contenidos se imparten en Matemáticas II, que es una asignatura troncal del segundo curso de las titulaciones: Grado en Ingeniería Eléctrica, Grado en Ingeniería Electrónica Industrial y Automática y Grado en Ingeniería Mecánica. Tanto los contenidos como la metodología de esta asignatura han experimentado cambios importantes: los contenidos han pasado de tener un carácter más teórico a combinarse con las prácticas de laboratorio, en las que trabajamos con el apoyo del programa Mathematica de Wolfram (Wolfram Research, 2017).

Puesto que en la actualidad nuestros alumnos son nativos tecnológicos resulta natural plantearse de qué manera podemos introducir las nuevas tecnologías en las aulas para que éstas contribuyan a mejorar su motivación y consoliden su formación. En nuestra asignatura las prácticas se realizan en un laboratorio informático, entorno que consideramos óptimo para dedicar parte del tiempo a actividades que involucren más activamente al alumnado.

Estas reflexiones nos han llevado a participar en el Proyecto clase inversa de la UPV (PCI, 2017) y, por tanto, a diseñar nuevas actividades y estrategias para las clases de prácticas, de manera que los alumnos trabajen algunos contenidos de cada sesión (suficientemente accesibles a su nivel) fuera del aula y de manera autónoma. En nuestro caso, los alumnos aprenden de forma no presencial a través de la visualización de vídeos 'screencast' complementados con apuntes, mientras que otras actividades, como resolución de problemas, discusiones y debates, se realizan en el aula.

Nos resulta interesante aplicar la metodología de clase inversa en las prácticas de la asignatura, ya que los alumnos pueden utilizar fuera del aula el programa informático con el que vamos a trabajar, puesto que la UPV dispone de licencias docentes del programa Mathematica para la comunidad universitaria. El alumno cuenta con todo el material, tanto en formato de apuntes como en forma de vídeos, lo que le permite realizar una puesta a punto previa a cada sesión de prácticas. Esto conduce a que el profesor interactúe de forma más activa con los alumnos, pudiendo destinar parte del tiempo en el aula para aclarar las dudas que 
les hayan podido surgir en la previsualización del material, o bien profundizar en las distintas estrategias utilizadas en cada sesión. De esta forma, los roles de alumno y profesor cambian y, por tanto, estamos utilizando la metodología conocida como clase inversa (Lage et. al. 2000).

Esta metodología, también conocida en la literatura como 'flip education' o 'flipped classroom', permite convertir el aula en un lugar para resolver problemas, avanzar conceptos de forma colaborativa (Bergmann et. al. 2012), dándole así un valor añadido a la actividad del profesor. Por otro lado, sabemos que, en otras universidades, han sido desarrolladas experiencias en esta misma línea con buenos resultados dentro de nuestra materia (Yong et. al. 2015, Kinney 2016).

En nuestro caso, con esta metodología los estudiantes están más motivados para ir a clase y participan de forma activa en todas las actividades que se les propone. Por otra parte, sus opiniones sobre esta metodología son bastante positivas, aunque siempre existen alumnos reticentes al cambio.

\section{Objetivos}

Los objetivos generales que pretendemos alcanzar realizando esta experiencia son:

- Consolidar los resultados de aprendizaje de los alumnos de Matemáticas II adoptando la clase inversa como metodología en las prácticas de la asignatura.

- Conseguir que los alumnos alcancen las competencias trabajadas tras las acciones realizadas en cada práctica y, así, conseguir el aprendizaje del programa Mathematica en la materia que nos ocupa.

Para ello, los objetivos específicos que nos proponemos al iniciar esta nueva metodología son los siguientes:

- Crear una unidad de aprendizaje en la plataforma PoliformaT de la UPV donde estén diseñadas las prácticas que vamos a realizar a lo largo del curso.

- Elaborar material didáctico en forma de apuntes.

- Diseñar y grabar vídeos 'screencast' para que sean visualizados por el alumno con anterioridad a la clase presencial.

- Preparar tareas cortas que los alumnos deben realizar posteriormente a la visualización de los vídeos.

- Escoger una lista de ejercicios o cuestiones para trabajar en grupo en el aula de forma presencial.

- Dinamizar la sesión presencial para que los alumnos interactúen en el aula aportando diferentes opciones y alternativas a la resolución de los ejercicios propuestos en la puesta en común final.

- Preparar exámenes de respuesta múltiple para la evaluación individual de los alumnos.

2017, Universitat Politècnica de València 


\section{Desarrollo de la innovación}

El programa de la asignatura Matemáticas II corresponde a la materia: ecuaciones diferenciales, transformada de Laplace y sus aplicaciones. Esta asignatura consta de 6 créditos: 3 créditos teóricos, 1 crédito de prácticas de aula y 2 créditos de prácticas informáticas. Respecto a asignaturas similares de planes de estudio anteriores correspondientes a la formación de los Ingenieros Técnicos Industriales, se ha optado por dar un enfoque más práctico de estos contenidos al adaptarlos a las titulaciones de grado. Ahora priorizamos las técnicas que favorecen la utilización de los conceptos estudiados en diferentes campos, así como el uso de herramientas y paquetes matemáticos más afables y eficientes a la hora de realizar los problemas. El modelo adoptado para impartir la asignatura consiste en combinar la teoría con prácticas de aula y prácticas de laboratorio, en las que trabajamos con el apoyo del programa Mathematica.

Con la implantación de la clase inversa en las prácticas no sólo pretendemos que el alumno aprenda a manejar el programa Mathematica para resolver ecuaciones diferenciales, mediante comandos que encuentren las soluciones tanto analítica como numérica de este tipo de ecuaciones, sino que además confiamos en que desarrolle las competencias transversales: "Análisis y resolución de problemas" y "Pensamiento crítico" que le permitan discernir no sólo si el proceso aplicado para resolver un problema es el correcto, sino que también sea capaz de valorar si existen aspectos que se puedan mejorar en dicho proceso. Por otro lado, esperamos que esta metodología refuerce el uso del programa en la resolución de ejercicios aplicados a la ingeniería, trabajando de esta forma otras competencias muy importantes para estos graduados como: "Comprensión e integración" y "Aplicación y pensamiento práctico". Todas estas competencias transversales forman parte del catálogo que la UPV ha considerado que todos sus egresados deben tener al finalizar sus titulaciones tanto de grado, como de máster (UPV, 2017).

El introducir en nuestras prácticas metodologías más activas como la clase inversa, nos ha llevado a rediseñar contenidos específicos para cada una de las sesiones de prácticas. La elaboración de estos contenidos se ha realizado en dos etapas: en la primera se elaboró el material didáctico en forma de apuntes y guías de trabajo para facilitar que el alumno realizase correctamente los ejercicios planteados con la ayuda del software informático. En la segunda etapa, hemos grabado presentaciones en forma de vídeos, denominados "screencast", con ejemplos concretos que ayudarán al alumno a abordar los problemas que se plantearán en las siguientes sesiones de prácticas. Y, por último, hemos elaborado tareas participativas en el aula, que se utilizan para evaluar el trabajo realizado por los alumnos fuera y durante la clase.

El programa de prácticas de ordenador de la asignatura Matemáticas II se muestra a continuación:

- Práctica 1: Introducción al programa Matemática

- Práctica 2: Ecuaciones diferenciales de primer orden

- Práctica 3: Ecuaciones diferenciales de orden superior

- Práctica 4: Sistemas de ecuaciones diferenciales 
- Práctica 5: Métodos numéricos para ecuaciones diferenciales

- Práctica 6: Transformada de Laplace

Para desarrollar esta nueva metodología hemos elaborado un plan de trabajo que involucra tanto las actividades que se deben realizar antes y durante la sesión en el laboratorio, como una coordinación exhaustiva entre las clases teóricas y las clases prácticas de la asignatura.

La organización de los materiales disponibles en cada práctica, así como las actividades que deben realizar, se muestra a los alumnos a través de la plataforma educativa de la UPV, denominada PoliformaT, donde hemos vertebrado, a través de la herramienta Lessons disponible en ella, los pasos que los alumnos deben seguir para finalizar con éxito cada práctica. Por ejemplo, en el caso de la práctica 2: Ecuaciones diferenciales de primer orden, hemos preparado tres vídeos titulados:

- Ecuaciones diferenciales Exactas. Factor integrante

- $\quad$ Solución general de la ecuación diferencial de orden 1

- $\quad$ Problema de valor inicial. Solución particular

que los alumnos deben visualizar antes de entrar en el laboratorio. Los objetivos que los alumnos deberían alcanzar tras visualizar estos vídeos vienen indicados en forma de conclusiones en la última diapositiva de cada uno de ellos, como se muestra en la Fig. 1.

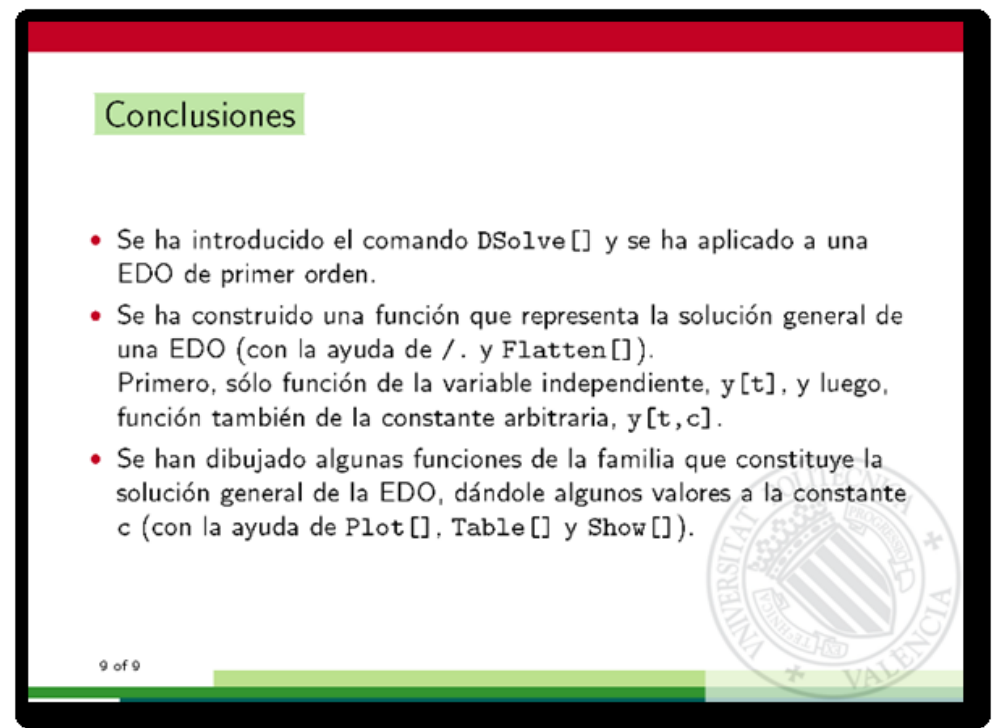

Fig. 1: Objetivos desarrollados en la práctica "Solución general de la ecuación diferencial de orden 1"

Para comprobar que efectivamente se han alcanzado dichos objetivos, las actividades en el laboratorio comienzan revisando los contenidos teóricos que se van a trabajar y planteando ejercicios relacionados con ellos. Los alumnos deben resolver los problemas en el aula, dejando el tiempo necesario para resolver todas las posibles dudas y preguntas que planteen al respecto, de manera que aprovechen este tiempo presencial para profundizar en los conceptos introducidos en los vídeos. En esta línea pretendemos que los "screencasts" ayuden al alumno a trabajar fuera del aula, a su propio ritmo y de forma más dinámica, los concep- 
tos que posteriormente se aplicarán en los problemas de las sesiones de prácticas de la asignatura.

Cada práctica se ha diseñado para ser realizada en dos sesiones consecutivas. En la primera sesión intentamos que el alumno tome conciencia de lo que ha aprendido y de las capacidades que ha alcanzado antes de enfrentarse a la evaluación definitiva de la práctica en la segunda sesión.

Para ello, al comenzar la clase hacemos al alumno unas preguntas relacionadas con los contenidos que se han introducido en los vídeos "screencast", sobre la práctica que vamos a realizar, a través de un test en PoliformaT. Con esto pretendemos confirmar que el alumno ha visualizado los vídeos y, por tanto, está preparado para trabajar los conceptos necesarios en la sesión.

Posteriormente se le plantean una serie de ejercicios, con dificultad creciente, para que compruebe por sí mismo si realmente ha entendido lo explicado y es capaz de aplicarlo a la resolución de estos problemas. Esta prueba puede realizarse individualmente o por grupos dependiendo de lo que el profesor considere más oportuno en cada sesión. En esta etapa el alumno puede preguntar todas las dudas que le vayan surgiendo a la hora de plantear o resolver los ejercicios, tanto al profesor que se encuentra dentro del aula, como a sus compañeros de grupo. Una vez finalizada la resolución de los ejercicios propuestos y antes de dar la solución correcta de los mismos se abre un debate donde cada grupo propone diferentes alternativas, aportando ideas para la resolución de los problemas, lo que a menudo genera interesantes debates sobre cuál sería la mejor solución. Finalmente, el trabajo realizado por cada alumno quedará registrado en un archivo, que se adjuntará a través de una tarea de PoliformaT, para que el profesor pueda supervisar en todo momento la labor realizada en cada sesión. Con estas actividades pretendemos que el alumno desarrolle la capacidad de análisis e integración de ideas y conceptos, así como la adaptación y aplicación de los mismos a la resolución de problemas relacionados con su especialidad.

En la segunda sesión de cada práctica evaluamos los conocimientos que han adquirido los alumnos. Para ello, realizamos en el aula una prueba individual donde tienen que resolver una serie de ejercicios relacionados con la materia que se ha trabajado en la sesión anterior. La calificación de estos ejercicios formará parte de la nota final de prácticas de la asignatura.

La evaluación de las prácticas se ha diseñado como una mezcla de pruebas objetivas, de elección múltiple, y problemas de desarrollo. Todas ellas están planteadas de forma que exigen a los alumnos la aplicación de principios, temas relacionados entre sí, interpretación de datos y resultados e incluso, en algunos casos concretos, emisión de juicios.

Finalmente, con el objetivo de evaluar la efectividad de esta metodología, hemos analizado los resultados de la encuesta que la UPV ha pasado de forma anónima a los alumnos al final del curso. En esta encuesta, totalmente independiente de la de evaluación del profesorado, se les pregunta sobre su opinión sobre la metodología de clase inversa, si ésta les parece adecuada para la asignatura Matemáticas II, así como si ha mejorado su percepción respecto a la adquisición de contenidos, la aplicabilidad de éstos en otras materias de su especialidad, etc. 
Las conclusiones de los resultados obtenidos se utilizarán para mejorar los planteamientos docentes en nuestra asignatura en cursos sucesivos.

\section{Resultados}

En la encuesta hemos constatado que con la aplicación de la metodología de clase inversa los estudiantes están más motivados para ir a clase y, participan de forma más activa en las actividades planteadas en el aula, con la que en su gran mayoría están de acuerdo. Por otra parte, sus opiniones sobre qué les ha parecido y cómo les ha afectado esta nueva metodología son bastante positivas, como vemos a continuación en los resultados de la encuesta que la UPV les ha pasado a los alumnos. En ella se les ha pedido que puntúen con una escala Likert, según el baremo de 1=Total desacuerdo hasta 5=Total acuerdo, las siguientes cuestiones:

- Con la aplicación de la metodología de clase inversa realizada en esta asignatura, muestra tu grado de acuerdo con las siguientes afirmaciones:

- He ido a clase con el trabajo previo realizado

- Ha contribuido a aumentar mi implicación en la asignatura

- Me ha resultado gratificante

- Ha requerido mucho tiempo por mi parte

- Ha permitido que el profesor dispusiera de más tiempo en clase para aclarar dudas

- Ha fomentado el trabajo en grupo

- Me ha ayudado a llegar mejor preparado a los exámenes

- Lo que más te ha gustado de la aplicación de la metodología de clase inversa realizada en esta asignatura ha sido:

- Los materiales facilitados por el profesor (vídeos y documentos utilizados)

- El trabajo realizado en clase (actividades de aula)

- El trabajo realizado en casa necesario para seguir las clases

- La relación con el profesor, más cercana y de trabajo conjunto

- La relación con mis compañeros

- El sistema de evaluación empleado

- La preparación de la asignatura por parte del profesor: todo organizado y planificado

- Valoración General

- Estoy muy satisfecho con esta experiencia/metodología

- Recomendaría esta experiencia/metodología a mis compañeros

- Me gustaría que esta metodología se aplicara al resto de asignaturas 
Comentamos a continuación los resultados más significativos sobre las opiniones de los alumnos de cada uno de los tres grados de la ETSID donde se ha aplicado la metodología de clase inversa.

\section{Grado en Ingeniería Eléctrica}

Los alumnos del Grado en Ingeniería Eléctrica han sido los más participativos a la hora de contestar la encuesta, ya que aproximadamente un $66 \%$ de los alumnos que la han utilizado en sus prácticas han opinado sobre ella. También han sido a los que más les ha gustado la experiencia, ya que han otorgado puntuaciones positivas de 3, 4 o 5 en un 95,5\% a estar muy satisfecho con ella y en un $91 \%$ a recomendarla a sus compañeros. Además, en un significativo porcentaje del 86,2\% les gustaría aplicarla al resto de asignaturas (Fig. 2).

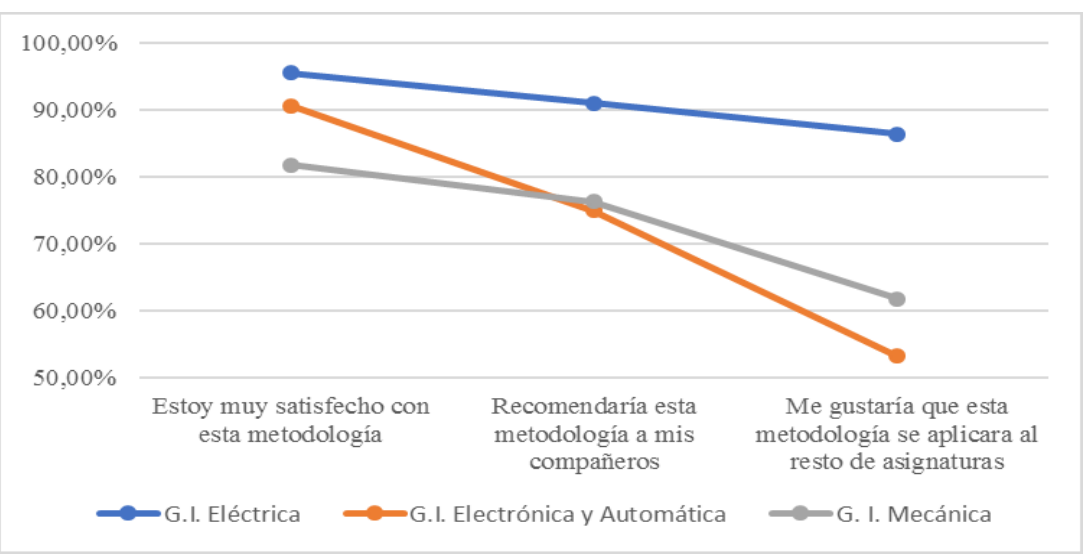

Fig. 2: Valoración general de la metodología clase inversa por parte de los alumnos de los distintos Grados

Algunos de los apartados de la encuesta más relacionados con la motivación y el aprendizaje han obtenido resultados muy favorables entre los alumnos de este grado. Por ejemplo, en el ítem "Ha contribuido a aumentar mi implicación en la asignatura" las respuestas positivas (puntuaciones de 3 a 5) alcanzan el 90,5\%, así como en el apartado "Ha permitido que el profesor dispusiera de más tiempo en clase para aclarar dudas" que llegan al 95,5\% y la afirmación "Me ha ayudado a llegar mejor preparado a los exámenes" que obtiene un $95,4 \%$ de respuestas positivas o muy positivas (Fig. 3). 


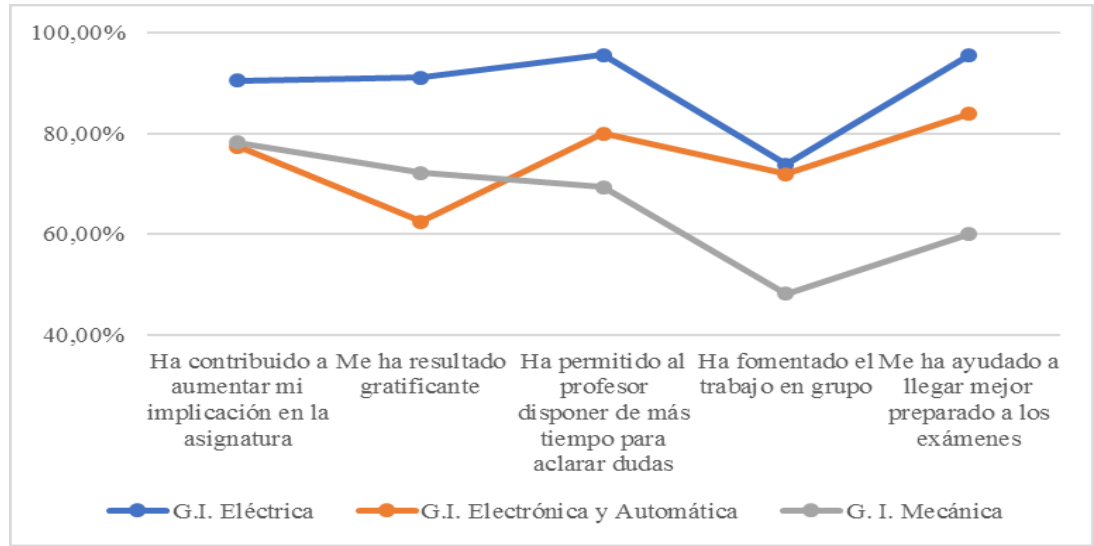

Fig. 3: Satisfacción de los alumnos de los distintos Grados con las afirmaciones relativas a la clase inversa

Los apartados relacionados con la interacción con el profesor y el resto de compañeros también salen muy bien parados, ya que el ítem "La relación con el profesor, más cercana $y$ de trabajo conjunto" obtiene un $99,9 \%$ de respuestas positivas o muy positivas y al $100 \%$ de los alumnos les ha gustado "La relación con mis compañeros", "El trabajo realizado en clase (actividades de aula)" y "El sistema de evaluación empleado". Por otro lado, estos alumnos valoran también muy positivamente la organización y planificación de la asignatura por parte del profesor (Fig. 4).

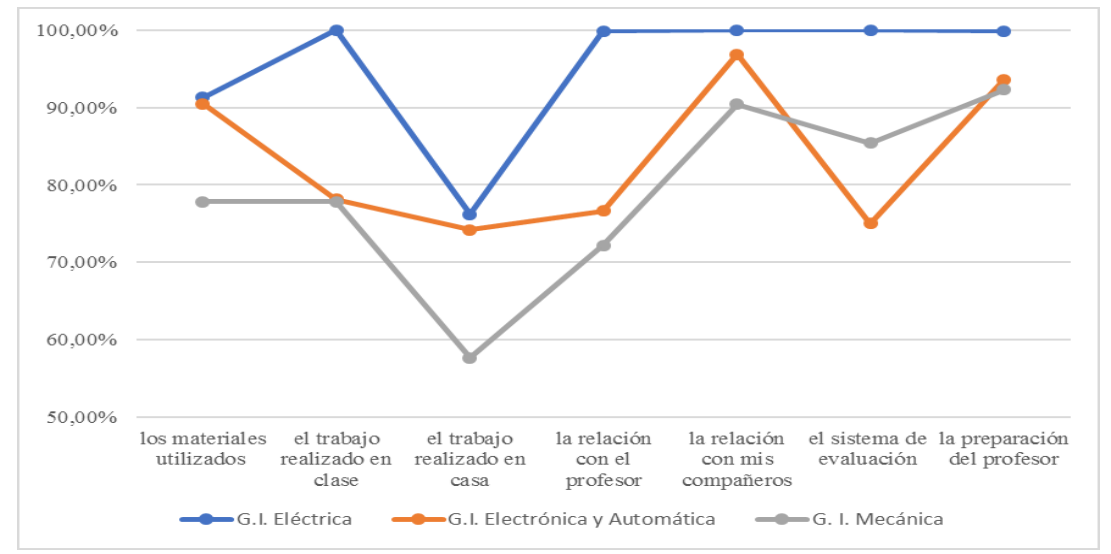

Fig. 4: Aspectos más favorables de la experiencia de clase inversa para los alumnos de los distintos Grados

Además, esta nueva metodología no les supone una carga de trabajo extra, ya que sólo un $18 \%$ de los alumnos opinan, con puntuaciones altas de 4 a 5 , que "Ha requerido mucho tiempo por mi parte", aunque sólo un $50 \%$ reconoce haber ido a clase con el trabajo previo realizado (Fig 5). 


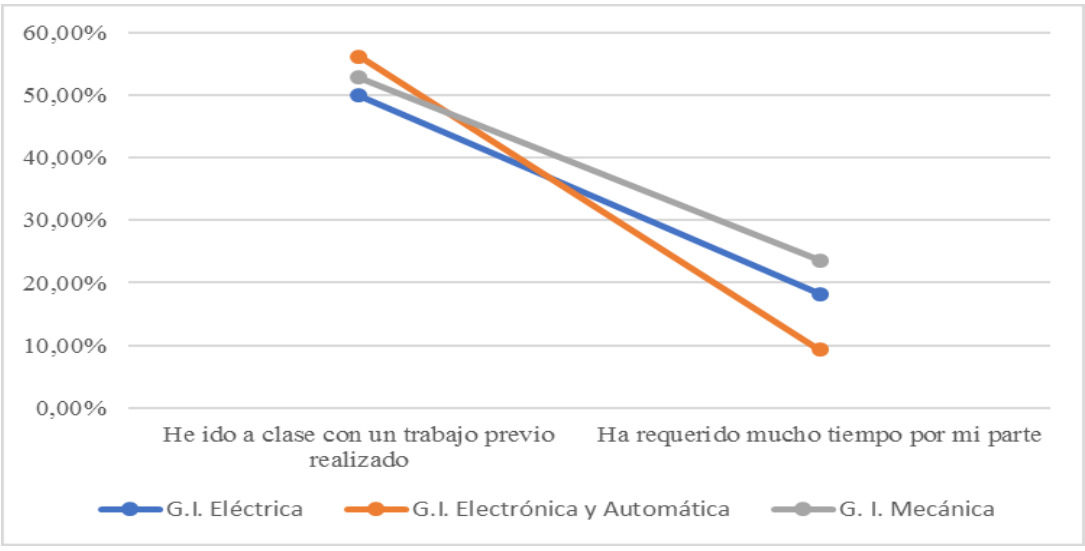

Fig. 5: Opiniones sobre el tiempo invertido en la nueva metodología por los alumnos de los diferentes grados

\section{Grado en Ingeniería Electrónica Industrial y Automática}

Aunque los alumnos del Grado en Ingeniería Industrial y Automática han sido los menos participativos a la hora de contestar la encuesta, sólo un $20 \%$ ha respondido las cuestiones planteadas, han sido, sin embargo, los que más comentarios libres nos han aportado al respecto. Las puntuaciones positivas ( 3 a 5 ) en el ítem "Estoy muy satisfecho con esta metodología" alcanzan un porcentaje del $90,5 \%$ en este caso y casi un $75 \%$ de los alumnos están de acuerdo en recomendarla a otros compañeros, aunque el porcentaje de aplicarla al resto de asignaturas baja a un 53,2\% en este grado (Fig. 2).

En el apartado de la encuesta relacionado con la mayor implicación en la asignatura los porcentajes positivos (puntuaciones de 3 a 5) rozan el 77,4\% y en el ítem sobre llegar mejor preparado a los exámenes alcanzan el 83,9\% (Fig. 3).

En este caso "Los materiales facilitados por el profesor (vídeos y documentos utilizados)" también han gustado al 90,5\% de los alumnos y en los ítems sobre la interacción destaca la relación con el resto de compañeros que alcanza un 96,9\%, con puntuaciones de 3 a 5, así como "La preparación de la asignatura por parte del profesor: todo organizado y planificado" que obtiene un 93,6\% de alta satisfacción entre los alumnos (Fig. 4).

En los alumnos de este grado, la percepción de sobrecarga ha sido menor ya que sólo un 9,3\% de los alumnos, opinan con puntuaciones altas de 4 a 5, que esta metodología ha requerido mucho tiempo por su parte, además de que un mayor porcentaje de alumnos, que ronde el 56\%, reconoce haber ido a clase con el trabajo previo realizado (Fig. 5).

Los comentarios libres que realizan estos alumnos se refieren principalmente al hecho de que la extensión de esta metodología al resto de asignaturas no les parece buena idea, como podemos ver en sus afirmaciones: "No en todas las asignaturas sería factible esta metodología", "Para esta asignatura este método es muy correcto, pero no estoy segura de si este método sería muy buena idea para otras asignaturas". Aunque también nos hacen sugerencias a la hora de plantear las actividades: "Sería ideal hacer un proyecto conjunto donde se puedan practicar los conocimientos adquiridos en las prácticas y combinarlos en las diferentes disciplinas de la ingeniería", así como de evaluar las tareas propuestas: "No me 
gusta que haya un examen final. Creo que haber realizado todas las prácticas y su trabajo previo, aparte de asistir a las clases, debería ser suficiente".

\section{Grado en Ingeniería Mecánica.}

En el Grado en Ingeniería Mecánica han respondido las preguntas de la encuesta aproximadamente un $40 \%$ de los alumnos. En este caso, las puntuaciones de 3 a 5 en el ítem "Estoy muy satisfecho con esta metodología" alcanza un porcentaje del $81 \%$ y un $76 \%$ el apartado sobre recomendar esta metodología a sus compañeros, aunque el porcentaje de aplicarla al resto de asignaturas, al igual que en los grados anteriores, baja a un 61,8\% (Fig. 2).

En este caso, en los apartados relacionados con la motivación sigue destacando el ítem sobre la mejora en la implicación de la asignatura, donde las puntuaciones de 3 a 5 alcanzan un porcentaje del $78,2 \%$, aunque aquí también desataca el ítem "Me ha resultado gratificante" que alcanza un $72,2 \%$ (Fig. 3 ).

En los apartados relacionados con la interacción destaca, como en los otros grados, la relación con el resto de compañeros, así como la preparación de la asignatura por parte del profesor, que alcanzan un $92,3 \%$ y un $90 \%$ de puntuaciones de 3 a 5 entre los alumnos, respectivamente (Fig. 4).

La percepción de sobrecarga aquí ha sido mayor que en el resto de grados, un 23,6\% de los alumnos opinan, con puntuaciones de 4 a 5 , que es esta metodología ha requerido mucho tiempo por su parte, aunque en el porcentaje de alumnos que reconoce haber ido a clase con el trabajo previo realizado, que en este caso es del $52,8 \%$, no hay mucha diferencia con el resto de grados (Fig. 5).

\section{Conclusiones}

Del análisis de las respuestas obtenidas en la encuesta, podemos concluir que aplicando la metodología de la clase inversa hemos mejorado en los dos principales objetivos que nos habíamos propuesto: aumentar la motivación de los alumnos y mejorar su aprendizaje significativo. Por otro lado, hemos conseguido aumentar y mejorar la relación con el profesor y el resto de compañeros que, desde nuestro punto de vista, también es muy importante para generar un buen ambiente en el aula.

Por tanto, consideramos la experiencia muy satisfactoria y estamos motivados para mejorar las carencias detectadas, trabajando en cursos sucesivos para mejorar las tareas planteadas, priorizando la modelización de problemas multidisciplinares e intentando que serán lo más realistas e integrados en el área profesional de los futuros graduados que nos sea posible. Aunque es importante darse cuenta que nuestra asignatura se encuentra en segundo curso y la formación de los alumnos todavía no es suficiente para abordar problemas muy específicos de la titulación que están cursando. 


\section{Referencias}

BERGMANN, J. and SAMS, A. (2012). Flip Your Classroom: Talk to Every Student in Every Class Every Day. International Society for Technology in Education. ISBN 9781564843159.

KINNEY, M. W. (2016). "Teaching an Online Sophomore-Level Differential Equations Course with Mathematica Supplements". CODEE Journal, Vol. 11, Article 1.

LAGE, M. J., PLATTl, G. J. and TREGLIA, M. (2000). "Inverting the classroom: A gateway to creating an inclusive learning environment". The Journal of Economic Education, 31 (1), p. 30-43.

PROYECTO CLASE INVERSA (2017) Universitat Politécnica de Valencia.

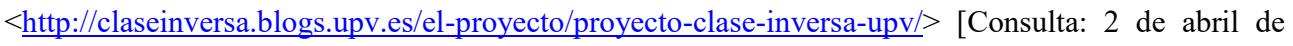
2017]

UNIVERSITAT POLITÈCNICA DE VALÈNCIA (2017). Proyecto institucional Incorporación de las competencias transversales en el currículo de los egresados de la UPV.

$<$ http://www.upv.es/entidades/ICE/info/U0724624.pdf $>$ [Consulta: 2 de abril de 2017]

WOLFRAM RESEARCH (2017). Mathematica de Wolfram.

$<$ http://www.wolfram.com/mathematica/?source=nav $>$ [Consulta: 2 de abril de 2017]

YONG, D., LEVY, R. and LAPE, N. (2015). "Why No Difference? A Controlled Flipped Classroom Study for an Introductory Differential Equations Course”, PRIMUS, 25 (9-10), p. 907-92. 